\title{
Does Dehydration alter the Success Rate and Technique of Three-Point Shooting in Elite Basketball? ${ }^{\dagger}$
}

Daniel Dinu 1,*, Eve Tiollier ${ }^{1}$, Elsa Leguy ${ }^{2}$, Marine Jacquet ${ }^{2}$, Jean Slawinski ${ }^{3}$ and Julien Louis ${ }^{4}$

1 French National Institute of Sport, Expertise and Performance, Sport, Expertise and Performance Lab., EA7370 Paris, France; eve.tioller@insep.fr

2 ESO Paris, Superior School of Osteopathy, 75012 Paris, France; elsaleguy@gmail.com (E.L.); jacquetmarine@sfr.fr (M.J.)

3 Centre de Recherche sur le Sport et le Mouvement (EA 2931), Université de Paris 10, UFR STAPS, 92000 Nanterre, France; jslawinski@u-paris10.fr

4 Research Institute for Sport and Exercise Sciences, Liverpool John Moores University, Liverpool L3 5UA, UK; J.B.Louis@ljmu.ac.uk

* Correspondence: daniel.dinu@insep.fr; Tel.: +33-1-4174-4177

+ Presented at the 12th Conference of the International Sports Engineering Association, Brisbane, Queensland, Australia, 26-29 March 2018.

Published: 13 February 2018

\begin{abstract}
In order to better inform the effects of dehydration and hydration guidelines, we tested the acute effects of a $2 \%$ dehydration protocol on performance and technique of 3-point shooting (3PS) in Elite Basketball players $(n=9)$. The 3PS technique was monitored by using nanotechnology inertial measurement units positioned onto body joints during the exercise. When dehydrated $(-2.1 \% \pm 0.5 \%$ of body mass $), 7$ players experienced a slight decrease $(-10.0 \% \pm 19.6 \%, p=0.16)$ in success rate, while RPE increased from $9.1 \pm 2.6$ to $13.0 \pm 2.5$ in euhydrated (EUH) and dehydrated (DEH) condition respectively $(p=0.003)$. DEH slightly altered the 3PS technique as the knee angle increased $(p=0.02)$ at toe-off time and the hip angle decreased during the 3PS $(p=0.01)$. The speed of ball release tended to be increased $(p=0.05)$ in DEH compared to EUH. Data from this study suggest that a $2 \%$ dehydration is tolerable for elite Basketball players, although a stronger dehydration might accentuate the effects observed.
\end{abstract}

Keywords: biomechanics; nanotechnology inertial measurement units; body kinematics; performance

\section{Introduction}

Basketball play is classically accompanied by a significant loss of body water with a potential negative impact on both physical and cognitive performance [1,2]. Osterberg et al. [3] reported that professional basketball players reached an average dehydration level of $1.4 \%$ per game. In addition, it is reported that basketball players often start games with a low hydration level accentuating the risk of dehydration in game and the negative impact on performance. Indeed, Osterberg et al. [4] reported that approximatively half of NBA players were dehydrated ( $\geq 1 \%$ of total body mass) before games during the seasons 2004 and 2005, and only $\sim 40 \%$ of water losses were replaced thanks to voluntary rehydration in game. In these conditions, the dehydration level was accentuated to -3 to $-4 \%$ of total body mass $[4,5]$. Considering that the final outcome of the game in basketball is often played within the last minutes of play, the impact of dehydration (low to high levels) on the ability to score could be determinant. 
Very few studies have examined the impact of dehydration on biomechanical adjustments required to score in basketball. Joints angles, the position of the centre of mass and ball release parameters (i.e., height, release speed and angle) are among the best predictive factors of success in basketball shooting [6,7]. The optimisation of these parameters is thus a prerequisite for high level basketball players looking for shooting accuracy and consistency, especially for three-point shots (3PS) which represent the highest score earned in basketball and generally generate $16 \%$ of points scored during a match. However, only 35\% of 3PS are successful in game likely due to the high complexity of the task, a strong opposition and/or fatigue mechanisms. Fatigue in match may alter the biomechanical requirements of successful 3PS and thus reduce the success rate [8]. Although results are still equivocal, fatigue might significantly alter shoulder and elbow joint angles and reduce the maximal height of the centre of mass, reducing in consequence the ball release height [8]. According to these data it can be hypothesized that dehydration might lead to similar alterations of the 3PS technique and even accentuate the effects of fatigue in game.

Within this framework, the purpose of the present study was to analyse the effects of a controlled dehydration protocol on the success and technique of 3PS in elite basketball players. Changes in shooting technique were analysed through changes in body kinematics (i.e., 3D angles of the ankle, knee, hip, shoulder, elbow and wrist, and the height of the centre of mass) and ball release parameters (i.e., height, velocity and angle of the ball at release) of 3PS in a dynamic playing condition.

\section{Materials and Methods}

\subsection{Participants}

Nine male basketball players (age: $16.2 \pm 0.7$ years; height: $1.97 \pm 0.07 \mathrm{~m}$; body mass: $87.7 \pm 8 \mathrm{~kg}$ ) volunteered to participate in this study. All participants were affiliated to the U18 squad of the basketball academy of the National team. This study was approved by the French National Basketball Association and was carried out in accordance with the Declaration of Helsinki. All participants were informed of the objectives and risks of the study and signed a consent form before participating.

\subsection{Study Design}

Each participant completed two trials scheduled at least one week apart, in a random order, either euhydrated (EUH) or dehydrated (DEH, $-2 \%$ of body mass). Upon arrival at the laboratory, participants completed the hydration protocol in order to achieve the desire level of hydration (EUH or DEH). After $10 \mathrm{~min}$ of rest and equipment with nanotechnology inertial measurement units, participants undertook the basketball protocol consisting of a series of standard basketball exercises. The imposed level of hydration was maintained throughout the whole basketball protocol.

\subsection{Euhydration/Dehydration Protocol}

Upon arrival at the laboratory, nude body mass was measured using a digital platform scale (SECA 803, accuracy $50 \mathrm{~g}$, Hamburg, Germany). Participants then entered an environmental chamber (Thermo Training Room, Paris, France) set at $39^{\circ} \mathrm{C}, 20 \%$ relative humidity and exercised on a cycle ergometer (Wattbike, West Bridgford, UK) for $60 \mathrm{~min}$ at light intensity $(90 \pm 10 \mathrm{~W})$. Depending on the trial, either no fluid (DEH trial) or water (EUH trial) was provided regularly throughout the cycling exercise in order to maintain the initial body mass or reach and maintain a $2 \%$ dehydration status. This procedure was repeated at the end of the $60 \mathrm{~min}$ exercise period to ensure that all participants maintained their body mass (EUH trial) or reached a 2\% dehydration (DEH trial).

\subsection{Basketball Protocol}

For both sessions, immediately after the hydration/dehydration protocol, participants were equipped with the MVN Biomech suit (Xsens Technologies BV, Enschede, The Netherlands) in order to track and record the upper and lower joint kinematics (i.e., 3D angles of the ankle, knee, hip, shoulder, elbow and wrist, and the height of the centre of mass) during the entire basketball protocol. 
The participants wore the MVN Biomech capture suit which also included a wireless data link. The sampling frequency was equal to $120 \mathrm{~Hz}$. After calibration of the MVN Biomech suit and habitual warm-up consisting of a series of 3PS in static and dynamic conditions, each participant was instructed to perform the maximum possible number of 3PS in $1 \mathrm{~min}$. An investigator recorded the number of successful and missed 3PS in $1 \mathrm{~min}$, as well as the rate of perceived exertion (RPE) at the end of the basketball protocol [9].

\subsection{Data Analysis}

\subsubsection{Kinematic and Ball Release Parameters}

The maximal and minimal positions of the centre of mass (CoM) were identified in order to calculate its vertical displacement. Then, ankle, knee, hip, shoulder, elbow and wrist angles for abduction/adduction, internal/external and flexion/extension angles of the joints on the side of the shooter were computed between the time when the CoM was at its lowest position (CoMmin), at toeoff time (Toe-off) and the time when the ball was released (CoMmax) (Figure 1). Ball release parameters (speed, angle and height at ball release) were also estimated. Kinematic analysis was performed with the software provided by MVN Biomech and a customized MatLab ${ }^{\mathrm{TM}}$ software was used to calculate the elbow, trunk, knee, and ankle joint angles.

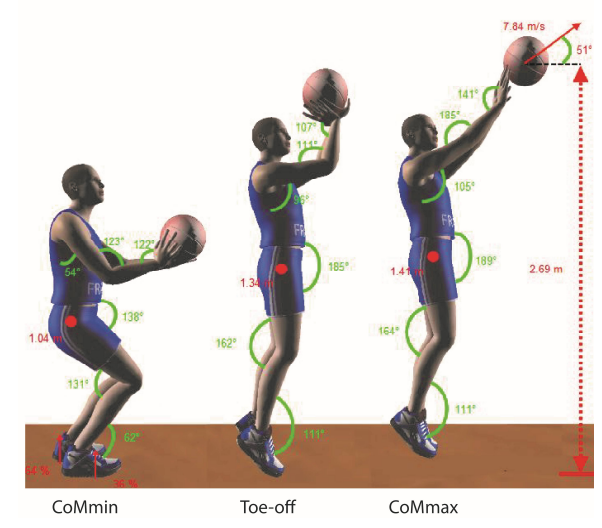

Figure 1. Joint angle orientations (green lines) for flexion/extension of all body joints and the position of the centre of mass (red dot) at the key time points of the 3PS.

\subsubsection{Statistical Analyses}

Differences between EUH and DEH were analysed by student t-tests for paired samples. When the assumption of normal distribution was violated, a non-parametric Wilcoxon signed-rank test was used. Effect sizes were also calculated using Cohen's $d$ values, with values $\leq 0.2,0.5,0.8$, and $>0.8$ considered as trivial, small, medium and large effects, respectively. For all statistical analyses, a $p$ value of 0.05 was considered to indicate significance. All data are presented as means \pm standard deviations (SD), unless otherwise indicated.

\section{Results}

\subsection{Hydration Status}

In the DEH condition, all players started the basketball protocol with a lower $(p=0.00, d=0.24)$ body mass $(-1.9 \mathrm{~kg} \pm 0.5 \mathrm{~kg}$ corresponding to $-2.1 \pm 0.5 \%$ of body mass $)$.

\subsection{Performance in Three-Point Shooting and Perceived Exertion}

The success rate tended to decrease $(p=0.16, d=0.65)$ for 7 players out of 9 following the dehydration protocol, from $51.2 \% \pm 12.2 \%$ to $41.3 \% \pm 18.3 \%$ in EUH and DEH conditions, respectively 
(Figure 2). The number of throws per minute was not different ( $p=0.78, d=0.14$ ) between DEH (10.7 $\pm 0.9)$ and EUH $(10.8 \pm 0.7)$ conditions, respectively (Figure 2).

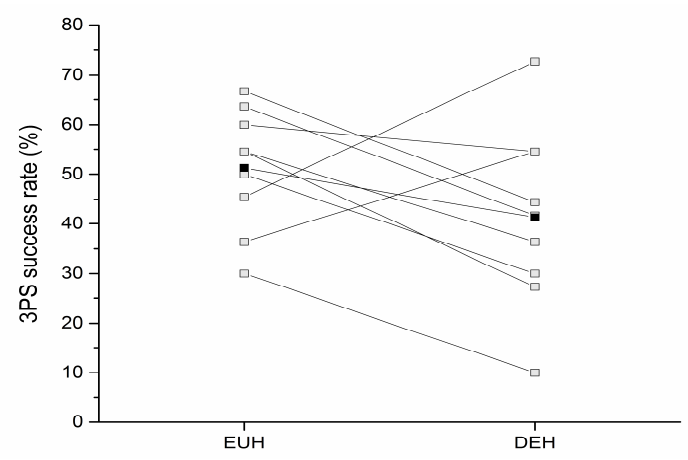

Figure 2. Mean (black dots) and individual (white dots) 3PS success rate in the EUH and DEH conditions.

The rate of perceived exertion (RPE) at the end of the basketball protocol was significantly greater $(p=0.003, d=1.50)$ in DEH condition $(13.0 \pm 2.5)$ compared to EUH condition $(9.1 \pm 2.6)$.

\subsection{Technical Aspects of Three-Point Shooting}

At toe-off time, the knee angle was significantly increased $(p=0.02, d=0.23)$ when dehydrated (DEH: $174.3 \pm 5.5^{\circ}$ ) compared to euhydrated (EUH: $173.1 \pm 5.2^{\circ}$ ). The hip angle between CoMmax and CoMmin was also significantly lowered $(p=0.01, d=0.60)$ in DEH condition $\left(38.7 \pm 12.0^{\circ}\right)$ compared to EUH condition $\left(45.1 \pm 8.5^{\circ}\right.$, Figure 3$)$. The position of CoM was not altered by dehydration at three key moments of 3PS. Only the speed of ball release tended to be increased $(p=0.05, d=0.53)$ in DEH condition $\left(7.82 \pm 0.12 \mathrm{~m} . \mathrm{s}^{-1}\right)$ compared to EUH condition $\left(7.76 \pm 0.13 \mathrm{~m} . \mathrm{s}^{-1}\right)$.
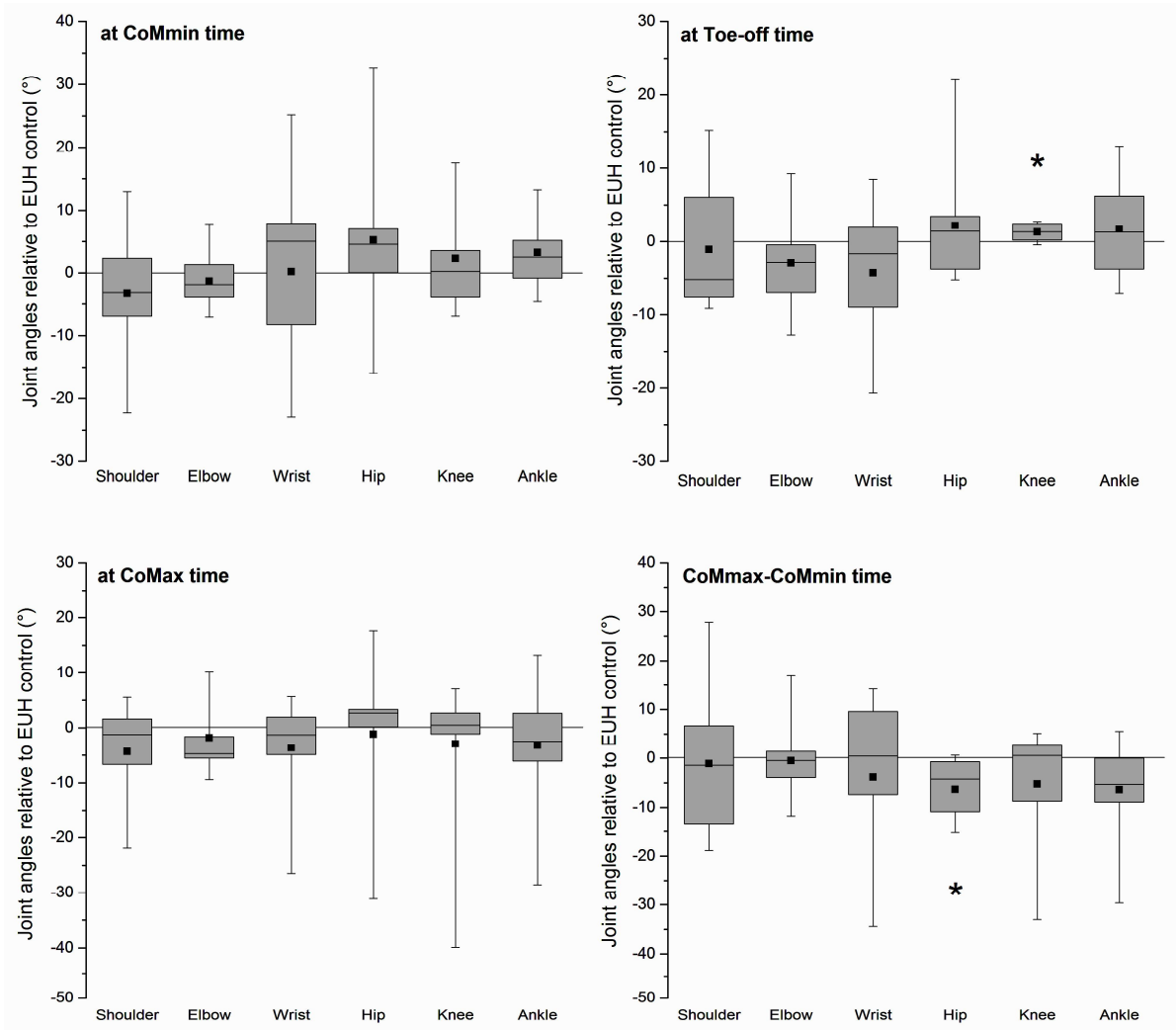

Figure 3. Box plots of joint angles changes at key time points of 3PS in DEH relative to EUH condition. The black square near the centre of each box represents the mean difference from EUH for each time point. * denotes a significant difference between EUH and DEH $(p<0.05)$. 


\section{Discussion}

Dehydration is often reported as a potential factor altering the accuracy of 3PS in Basketball. Considering the importance of 3PS in the final score in Basketball game, this study investigated for the first time the influence of a controlled $2 \%$ dehydration protocol on performance and technique of 3PS in Elite basketball players. The main results indicate a slight but non-significant decrease of performance in 3PS with a $2 \%$ dehydration status, accompanied with minor changes of body kinematics and ball release variables.

The mean decrease of body mass obtained at the end of the heat-induced dehydration protocol was $2.1 \% \pm 0.5 \%$, whereas it was only $-0.5 \% \pm 0.3 \%$ in the EUH protocol, the latter corresponding to euhydration in hot environments [10]. A reduction of $2 \%$ of body mass was targeted in the dehydration protocol in order to replicate the level of dehydration classically observed in basketball [3] and because the majority of the literature reports that body water deficits equivalent to 2\% body mass may impair physical and cognitive performance [1,2,11]. For example, Hoffman et al. [11] recorded a 19\% reduction of anaerobic power in a 2 on 2 full-court basketball game with dehydrated 17 -year-old players ( $-1.9 \%$ of body weight). In the present study, contrarily to previous studies, the $2 \%$ dehydration protocol had only a minor impact on performance in a 1-min dynamic shooting test. The success rate slightly decreased after the dehydration protocol compared to euhydration (from $51.2 \% \pm 12.2 \%$ to $41.3 \% \pm 18.3 \%$, NS). The inconsistencies between studies could be explained by the difference between exercise durations, i.e., around $80 \mathrm{~min}$ of basketball games in Dougherty et al. [2] and Baker et al. [1] studies whereas it was only series of 1-min shooting sessions in the present study. In addition to dehydration, the longer basketball protocols had likely depleted glycogen stores to a greater extent than in the present study, contributing to a significant impairment in basketball performance. Another possible explanation relies on the different training levels of players, i.e., elite members of the national team in the present study vs. non-elite in other studies, suggesting that basketball skills might be more robust to dehydration in elite players compared to non-elite.

Similar to performance results, the biomechanical analysis revealed minor effects of the $2 \%$ dehydration protocol on any of the kinematic parameters measured or estimated during the 3PS. Only the knee angle at toe-off time was greater when dehydrated while the hip angle was lower during the whole 3PS, without any alteration of the CoM position. These results suggest that the body kinematics engaged to perform a 3PS are robust enough in elite basketball players to maintain their ability to score despite a $2 \%$ dehydration. Similarly, several studies have reported a greater consistency of kinematic patterns of free throw shooters who had more playing experience [12,13]. More recently, Verhoeven et al. [14] recently showed that among 25 college basketball players repeating 50 three throws, the poor shooters presented the largest CoM trajectory variations between shots, compared to the best shooters. Another possible hypothesis to explain the absence of any alteration of the shooting technique is that our players were probably used to mild dehydration $(\sim 1-$ $2 \%$ body mass loss). Indeed, it is classically reported that basketball players experience episodes of dehydration on a regular basis due the combination of warm playing environments and chronic hypohydration [3,5].

Ball release variables were also investigated because they are the ultimate determinants of shot success, regardless of shooting posture. Only the speed of ball release tended to be greater when dehydrated $(p=0.05, d=0.6)$. Although, it is well accepted that a high ball release allows a lower movement velocity and release angle, leading to a better accuracy [6,15], recent findings suggest that ball release parameters would not necessarily rely on specific patterns automated through practice. On contrary, ball release parameters would rather be highly variable and adaptable to the position of the body segments at shooting time [14]. According to these data, variance in ball release parameters might reflect a positive biomechanical adaptation in response to postural variance (i.e., knee and hip joint angles in the present study) to maintain the 3PS success rate.

\section{Conclusions}

The aim of this study was to assess the effects of a $2 \%$ dehydration on 3PS performance and technique in elite basketball players. Nanotechnology inertial measurement units were positioned 
directly onto body segments to provide a full decomposition of the biomechanical variables associated with the 3PS task. Results indicate minor and non-significant effects of a $2 \%$ dehydration on the 3PS success rate accompanied with slight alterations of body kinematics and ball release variables. These data suggest that a $2 \%$ dehydration represents a tolerable level of dehydration to maintain performance and technique of 3PS in elite basketball players.

Conflicts of Interest: All authors approved the final version of the article and all declare that they have no conflicts of interest in relation to the present study.

\section{References}

1. Baker, L.B.; Dougherty, K.A.; Chow, M.; Kenney, W.L. Progressive dehydration causes a progressive decline in basketball skill performance. Med. Sci. Sports Exerc. 2007, 39, 1114-1123.

2. Dougherty, K.A.; Baker, L.B.; Chow, M.; Kenney, W.L. Two percent dehydration impairs and six percent carbohydrate drink improves boys basketball skills. Med. Sci. Sports Exerc. 2006, 38, 1650-1658.

3. Osterberg, K.L.; Horswill, C.A.; Baker, L.B. Pregame urine specific gravity and fluid intake by National Basketball Association players during competition. J. Athl. Train. 2009, 44, 53-57.

4. Osterberg, K.L.; Sperber, T.; Lacambra, L.; Baker, L.B.; Murray, R. Fluid balance, hydration status, and sweat electrolyte concentrations in NBA basketball players during summer league games. J. Athl. Train. 2005, 40, S40.

5. Osterberg, K.L.; Horswill, C.A.; Sperber, T.; Tedeschi, F.; Murray, R. Fluid balance, hydration status, and sweat electrolyte concentrations in NBA basketball players during pre-season practice. Med. Sci. Sports Exerc. 2004, 36, S180.

6. Hamilton, G.R.; Reinschmidt, C. Optimal trajectory for the basketball free throw. J. Sports Sci. 1997, 15, 491504.

7. Okazaki, V.H.; Rodacki, A.L.; Satern, M.N. A review on the basketball jump shot. Sports Biomech. 2015, 14, 190-205.

8. Erculj, F.; Supej, M. Impact of fatigue on the position of the release arm and shoulder girdle over a longer shooting distance for an elite basketball player. J. Strength Cond. Res. 2009, 23, 1029-1036.

9. Borg, G.A. Psychophysical bases of perceived exertion. Med. Sci. Sports Exerc. 1982, 14, 377-381.

10. Greenleaf, J.E. Problem: thirst, drinking behavior, and involuntary dehydration. Med. Sci. Sports Exerc. 1992, $24,645-656$.

11. Hoffman, J.R.; Stavsky, H.; Falk, B. The effect of water restriction on anaerobic power and vertical jumping height in basketball players. Int. J. Sports Med. 1995, 16, 214-218.

12. Button, C.; MacLeod, M.; Sanders, R.; Coleman, S. Examining movement variability in the basketball freethrow action at different skill levels. Res. Q. Exerc. Sport 2003, 74, 257-269.

13. Okazaki, V.H.; Rodacki, A.L. Increased distance of shooting on basketball jump shot. J. Sports Sci. Med 2012, $11,231-237$.

14. Verhoeven, F.M.; Newell, K.M. Coordination and control of posture and ball release in basketball freethrow shooting. Hum. Mov. Sci. 2016, 49, 216-224.

15. Miller, S.; Bartlett, R. The relationship between basketball shooting kinematics, distance and playing position. J. Sports Sci. 1996, 14, 243-253.

(C) 2018 by the authors. Licensee MDPI, Basel, Switzerland. This article is an open access article distributed under the terms and conditions of the Creative Commons Attribution (CC BY) license (http://creativecommons.org/licenses/by/4.0/). 